\title{
The Future of Energy Markets and the Challenge of Decentralized Self-Management
}

\author{
Frances Brazier ${ }^{1}$ Elth Ogston ${ }^{2}$ Martijn Warnier ${ }^{1}$ \\ ${ }^{1}$ Intelligent Interactive Distributed Systems ${ }^{\star \star}$ \\ VU University, Amsterdam \\ The Netherlands \\ \{M.Warnier, FMT.Brazier\}@few.vu.nl \\ ${ }^{2}$ Department of Computer Science \\ University of Warwick \\ United Kingdom \\ elth@dcs.warwick.ac.uk
}

\begin{abstract}
Complex, intelligent, distributed systems in dynamic environments, such as the power grid need to be designed to adapt autonomously. Self-management, in particular of large scale adaptive systems such as the power grid, is necessarily distributed. Agent and peer-to-peer based decentralized self-management can change the future of energy markets in which the power grid plays a core role.

Assuming that both consumers and providers of energy are autonomous systems, represented by software agents or peers capable of self-management, virtual organizations of systems can emerge and adapt when necessary. Communication structures between systems, e.g., hierarchical or clustered organizations, can emerge, organizations between and within which systems can choose to cooperate and coordinate their actions, or compete. Overlay structures (as defined within $\mathrm{p} 2 \mathrm{p}$ research) define such adaptive communication structures, multi-agent research provides interaction patterns. Global goals are achieved by local management on the basis of local goals and knowledge. The appropriate delegation of managerial responsibility determines the control structure. Aggregate information differs depending on the position of a system in an organization, the aggregation mechanisms and policies.
\end{abstract}

\section{Introduction}

Complex, intelligent, distributed systems in large-scale dynamic environments need to be designed to adapt. Self-management, in particular of large scale adaptive systems such as the power grid, is necessarily distributed. Agent and

**As of September 12009 the IIDS group will be part of the Systems Engineering group, Faculty of Technology, Policy and Management, Delft University of Technology 
peer-to-peer based decentralized self-management can change the future of energy markets in which the power grid plays a core role. Agent and peer-to-peer based decentralized self-management can change the future of energy markets.

Energy markets contain vast numbers of systems/devices that produce and consume electricity. These systems/devices are distributed across many interconnected networks, both physical and organizational, each with their own local service requirements. They operate in a complex and ever changing physical environment, and must serve the needs of a highly autonomous user group.

Decentralized self-management is the approach this paper explores. Emergent virtual organizations with local knowledge are needed to structure, and thus reduce, the complexity of global management tasks. Within these organizations, interaction protocols are needed for systems to coordinate their individual behaviors.

The total power generated by a grid, for example, must be related to the total demand, taking constraints on the transport network into account. The better this management task is performed, the more efficient the system is as a whole. In the ideal case all systems connected to a grid coordinate their plans for energy production and use, continuously adapting to take into account the changing constraints imposed by their environment and users, with minimal overhead and performance loss.

Dynamic management on the scale of an electrical grid of smart devices raises a number of key challenges with respect to most effective virtual organizations:

\section{- Creating and maintaining multi-level dynamic virtual organizations:}

Communication structures

Overlay structures define connections between agents: communication networks. These networks need to adapt to change in their dynamic environment. They also need to be maintained. They define the potential paths for dissemination and aggregation of information. Communication structures include (1) hierarchical networks, (2) semi-clustered hierarchical networks, (3) clustered network, or (4) fully unstructured networks, as depicted below in Figures 1-4. Which network structure is most appropriate for which application under which conditions is an important research question. The value of multi-level communication structures with different communication structures at different levels within an organization for different purposes, is currently a topic of research. Other research questions include determining the ideal number of systems within each network, membership criteria of networks, and recognizing when group membership should change.

- Division of management responsibility within a multi-level dynamic virtual organization:

\section{Control}

Given one or more global goals, determining which appropriate local goals can be negotiated with/delegated to specific systems within an organization is a challenge. Which level of authority should be assigned to which network 


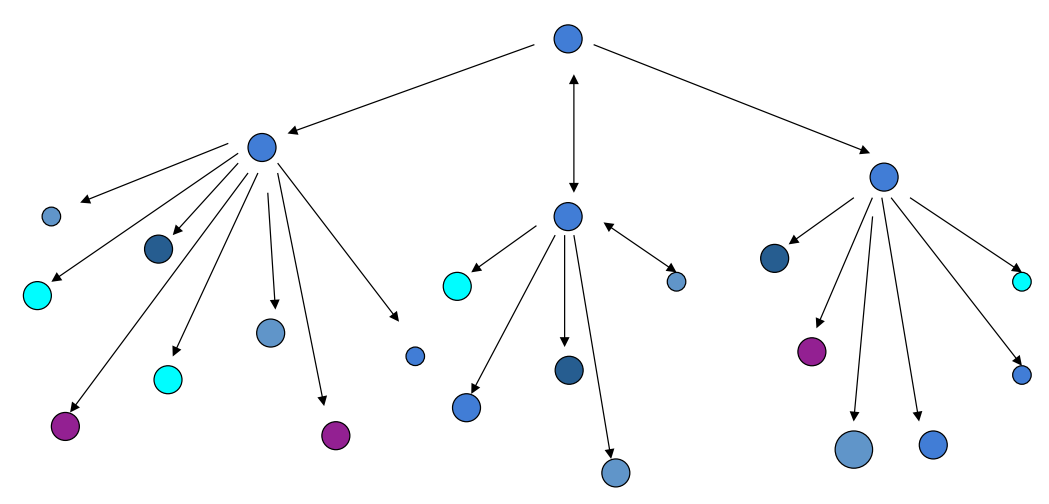

Figure 1. Hierarchical network structure

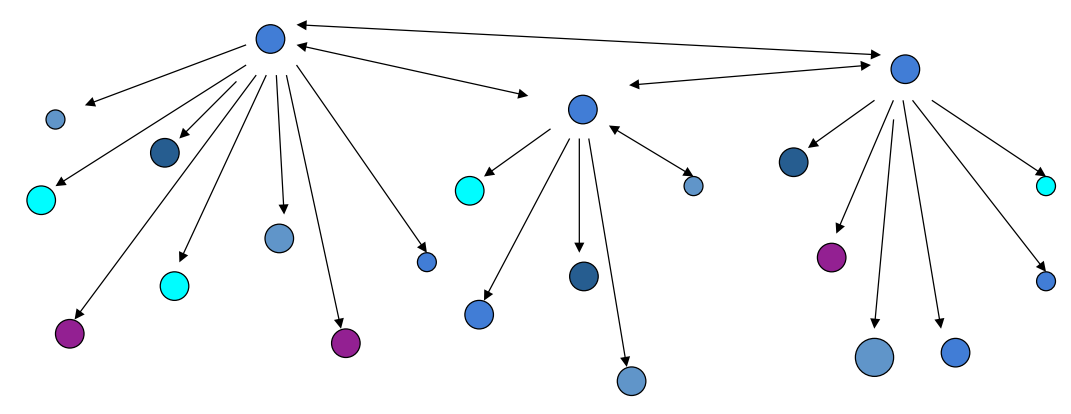

Figure 2. Semi-structured communication network

within a virtual organization? Which is most effective? Which systems can be assigned which level of authorization?

- Creating and maintaining multi-level dynamic virtual organizations: Aggregation of information

A system's location within a communication structure determines the information it can access and process, its local knowledge of system behavior. Aggregation policies and mechanisms define which information can be known where and when. Research questions include: What is the appropriate algorithm for aggregation of information? And how does simplifying or increasing the complexity of a management task at one level effect the complexity of management at other levels? 


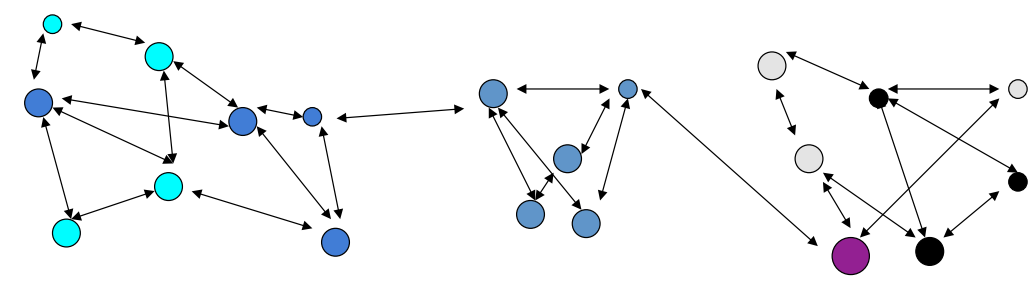

Figure 3. Clustered communication network

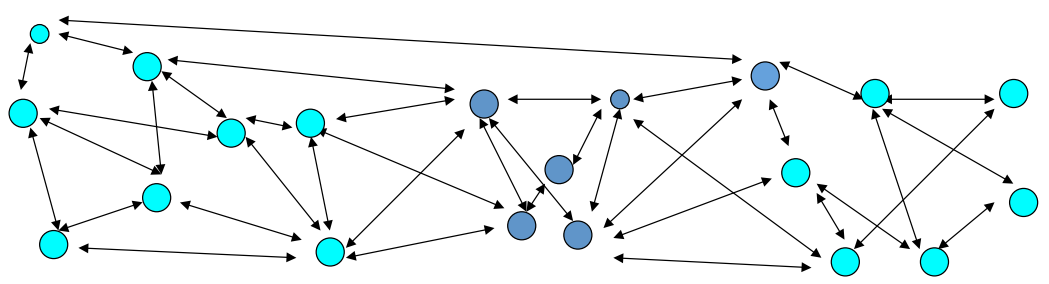

Figure 4. Unstructured network structures

\section{The Future of the Energy Market}

The scale and highly distributed nature of the power grid necessitates distribution of management. Virtual organizations are a means with which such distribution can be regulated: the communication structure, distribution of control, distribution of managerial responsibility.

Demand for electricity is, for the most part, although partially predictable, not controllable, and storage capacity is limited. Currently the only solution to fluctuations in demand, limited flexibility of large power plants, and strict global reliability requirements, is for providers to have more power produced than strictly necessary with a higher capacity than most often required.

Recent technological developments, however, are opening new possibilities for improving power grid management. Ubiquitous communications networks and low-cost controllers may provide the flexibility the market needs. Improvements in solar panels, domestic-sized wind turbines, and micro-CHP, and the increasingly widespread availability of high capacity batteries in devices such as electric vehicles change how and where power is most efficiently generated and stored. Current grid management systems are, however, limited in scale by their use of partially centralized decision making. Wholesale markets use a centralized clearinghouse to calculate and distribute prices and to inform participants of winning and losing bids. This means that only large power plants and utilities participate in electricity markets. To incorporate larger numbers of participants, 
further decentralization is required. Research on distributed electricity resource management (DER) addresses this problem[1,2,3], bringing together and furthering research on peer-to-peer and multi-agent systems.

\section{Challenges of Decentralized Self-Management}

Distributed energy resource management is a challenge: self management the solution proposed. This section highlights some of our research efforts in this area.

\subsection{Distributed self-management of individual appliances: communication and aggregation}

Virtual organizations of agents define communication structures between agents, e.g., hierarchical organizations [4], between and within which agents can choose to cooperate and coordinate their actions, or compete. Dynamic organized hierarchies [5] can be used to support adaptive, aggregate, nonlinear behavior, as a means to reduce complexity. Coordination in unstructured environments entails distributed search and distributed scheduling [6,7].

Our current research in this area focuses on the level of individual appliances. Global stabilization of the energy consumption of an electricity network (power grid), by minimizing oscillations of all thermostatic controlled appliances (TCA) within a single household, is the goal. Such devices, for example, refrigerators, air conditioners and water heaters, consume $25 \%$ of the total energy supply in the USA [8]. Self management of these devices could potentially have a significant effect on the stabilization of global resource consumption. To this purpose software agents in TCAs autonomously and automatically negotiate their resource requirements, configurations, and SLAs, ultimately acquiring global stabilization in energy consumption, which in turn leads to higher reliability of the power grid and lower costs for consumers and producers.

This study [9] proposes a fully decentralized agent-based approach to global stabilization of resource utilization, for energy consumption, based on local coordination. The approach is threefold and can be outlined as follows: (i) Agents are members of a hierarchical virtual organization, structuring agent interactions and aggregation of agent resource requirements. (ii) A simple agent knowledge model is assumed on the basis of which resource requests are generated. (iii) Agents can make local adaptive decisions on the basis of information they receive from the agents to which they are linked. This research focuses on minimizing the oscillations of thermostatic controlled appliances. Software agents autonomously negotiate their resource requirements and configuration $[10,11]$.

Using relatively straightforward models of energy devices this research [9] has shown that hierarchical local coordination achieves emerging convergence of the global stabilization through local knowledge, local decisions and local interactions by individual software agents. Self-management has thus shown to be feasible, as demonstrated in agent-based simulations, in the AgentScape [12] 


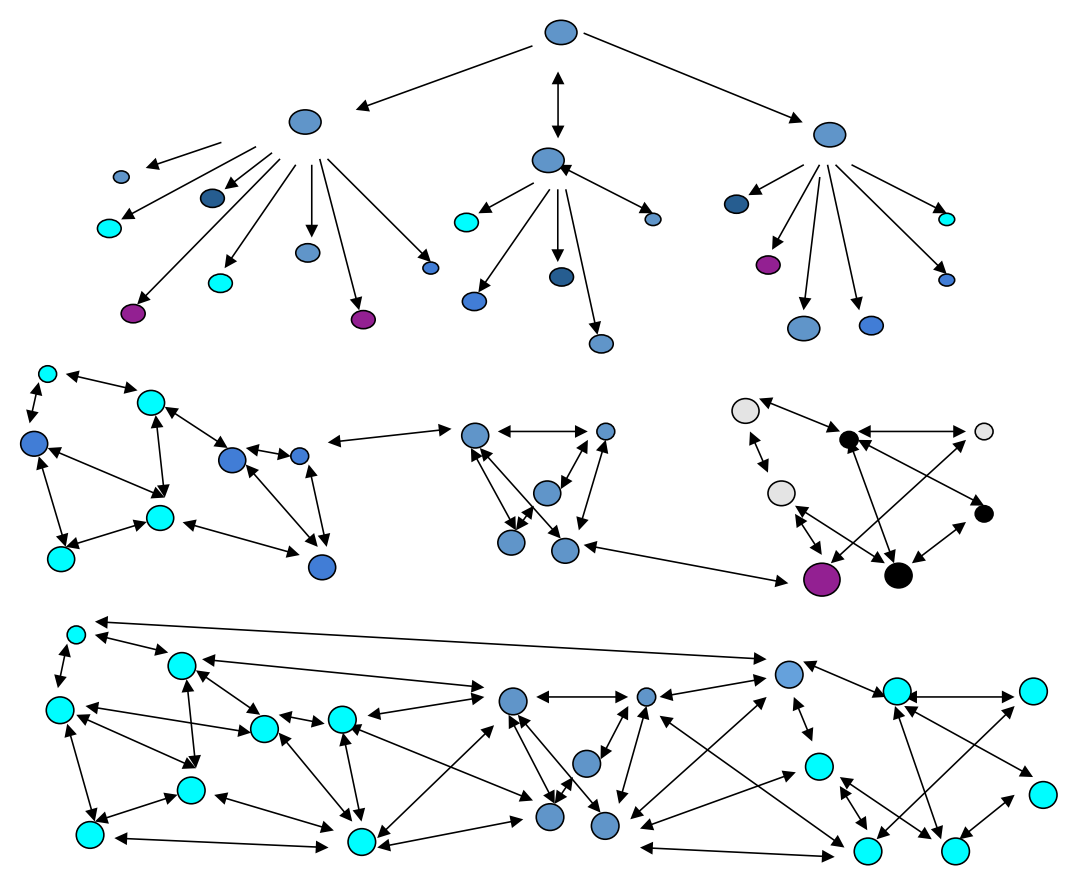

Figure 5. Layered network structures

platform. The hierarchical organization plays an important role in the proposed method. Current research therefore focuses on peer-to-peer based self-managed robust hierarchical topologies designed to adapt to local failures, reorganizing the topology as needed on the basis of local knowledge.

In [13] an approach for building such robust hierarchical topologies is investigated. The approach is based on a 3-layer communication overlay architecture for self-organization. Figure 5 depicts the approach. Communication between the different network layers (as one depends on the other) is not depicted, but is a essential to the approach.

\subsection{Self management in virtual power stations: managerial responsibility}

How management can be most effectively divided between parties in distributed energy resource management problems is another issue our research addresses. Virtual power stations consisting of a large number of nodes, such as office buildings, each with a small amount of generating capacity group together to form an entity that can participate in an electricity market $[1,14]$. Control agents on each node manage local resources and interact with each other to achieve global 
goals. In our research a scenario based on the measured output of a photovoltaic installation in Newcastle, NSW, Australia, and price data taken from the Australian National Electricity Market has been used to study different management options. The amount of profit that may be realized under different forms of distributed management in realistic operating conditions has been compared.

There are many ways in which a resource management task, like that encountered in DER and in this scenario, can be divided between locations. Methods can be classified by where final authority for resource use is located. Authority can lie with a central overseer, for instance as commonly used in computational grids to plan and allocate jobs so as to make optimal use of a set of processors. Alternatively, management can be decentralized, with each node having authority over its own resources, as is often the goal in multi-agent systems which aim to maximize node autonomy. Or central and local managers can have complimentary tasks, as in many peer-to-peer systems where a central controller manages the composition of mostly independent groups of nodes, but within groups nodes have control of their local resources.

Which of the above methods of apportioning control is most appropriate depends upon diverse application requirements, including the degree to which the desired behavior differs from the systems natural behavior, the sensitivity of the system to parameter variations, and the independence required by individual components in order to be able to accommodated local constraints $[15,16]$.

Understanding how various distributed management schemes influence achievable profit in a virtual power station is important because the form of distributed management employed has consequences for the required communication and computation infrastructure. Strong central coordination requires powerful servers. Frequent or precise coordination requires high capacity, reliable communication links. Advanced self-management techniques require system monitoring as well as complex devices on which to run local control agents. The benefit of a management scheme may therefore be outweighed by the cost of implementing it.

Simulation experiments testing the ways these forms of management can be combined to control a virtual power station have shown that each has an important role to play. However, the quality of management in each case is less important. Weak management techniques have shown to provide substantial increases in profit. Increasing the quality of management, on the other hand, only leads to small additional gains. However, a balanced combination of loosely coordinated basic controllers may be preferable.

These results indicate that DER could benefit from exploring the right balance of control in hybrid management systems, while efforts that focus only on perfecting control at a specific point or improving coordination between points may not produce cost effective solutions. 


\section{Conclusions}

The energy market is a complex, intelligent, distributed system in a dynamic environment that needs to adapt continually, and thus needs to be designed to this purpose. The multi-agent systems and peer-to-peer paradigms together provide a promising frame of reference for the design of autonomic self-managed configurations of systems for the power grid.

\section{Acknowledgments}

This project is supported by the NLnet Foundation http://www.nlnet.nl. The authors thank Evangelos Pournaras for his contributions to this paper.

\section{References}

1. James, G., Peng, W., Deng, K.: Managing Household Wind-energy Generation. Intelligent Systems, IEEE 23 (2008) 9-12

2. Ogston, E., Zeman, A., Prokopenko, M., G., J.: Clustering Distributed Energy Resources for Large-Scale Demand Management. In: Proceedings of the 1st Int. Conf. Self-Adaptive and Self- Organizing Systems (SASO07), IEEE (2007) 97-108

3. Varga, L.Z., Jennings, N.R., Cockburn, D.: Integrating Intelligent Systems in to a Cooperating Community for Electricity Distribution Management. International Journal ofExpert SystemswithApplications 7 (1994) 563-579

4. Jennings, N.R.: An agent-based approach for building complex software systems. Commun. ACM 44 (2001) 35-41

5. Luis, M.: Complex system modeling: Using metaphors from nature in simulation and scientific methods. BITS: Computer and Communications News, Computing, Information and Communications Divisions (1999)

6. Theocharopoulou, C., Partsakoulakis, I., Vouros, G.A., Stergiou, K.: Overlay networks for task allocation and coordination in dynamic large-scale networks of cooperative agents. In: AAMAS '07: Proceedings of the 6th international joint conference on Autonomous agents and multiagent systems, ACM (2007) 1-8

7. A., J., Davidsson, P., Carlsson, B.: Coordination models for dynamic resource allocation. In: Coordination Models and Languages. (2000) 182-197

8. Mazza, P.: The smart energy network: Electrical power for the 21st century. Climate Solutions (2002)

9. Pournaras, E., Warnier, M., Brazier, F.M.T.: A distributed agent-based approach to stabilization of global resource utilization. In: the International Conference on Complex, Intelligent and Software Intensive Systems (CISIS'09), IEEE (2009)

10. Hammerstrom, D.: Part II. Grid Friendly ${ }^{\text {TM }}$ Appliance Project. PNNL 17079, Pacific Northwestern National Laboratory (2002)

11. James, G., Cohen, D., Dodier, R., Platt, G., Palmer, D.: A deployed multi-agent framework for distributed energy applications. In: 5th International Joint Conference on Autonomous Agents and Multi-agent Systems (AAMAS 2006),Hakodate, Japan. (2006)

12. Overeinder, B.J., Brazier, F.M.T.: Scalable middleware environment for agentbased Internet applications. In: Applied Parallel Computing. Volume 3732 of Lecture Notes in Computer Science., Berlin, Springer (2006) 675-679 
13. Pournaras, E., Warnier, M., Brazier, F.M.T.: Adaptive Agent-based Selforganization for Robust Hierarchical Topologies. In: Proceedings of the First International Conference on Adaptive and Intelligent Systems (ICAIS'09), IEEE (2009) to appear.

14. Li, R., Wang, P.: Pattern Learning and Decision Making in a Photovoltaic system. In: Proceedings of the 7th International Conference on Simulated Evolution and Learning, (SEAL08). (2008) 483-492

15. Abu-Sharkh, S., Arnold, R., Kohler, J., Li, R., Markvart, T., Ross, J., Steemers, K., Wilson, P.and Yao, R.: Can Microgrids Make a Major Contribution to UK Energy Supply? Renewable and Sustainable Energy Reviews 2 (2006) 78-127

16. Hatziargyriou, N., Asano, H., Iravani, R., Marnay, C.: Microgrids. Power and Energy Magazine, IEEE 4 (2007) 78-94 